Hiroshi Umemura

Nagoya Math. J.

Vol. 42 (1971), 1-7

\title{
FORMAL MODULI FOR p-DIVISIBLE GROUPS
}

\section{HIROSHI UMEMURA}

The formal moduli for one-parameter formal Lie groups was constructed by Lubin and Tate (1) by using Lazard's methods. The aime of this paper is to prove the existence of the formal moduli for higher dimensional formal groups.

The author learned, after obtaining this, that P. Cartier established more general result. He was suggested that it might be worthwhile to publish this, Cartier's result notwithstanding, since our treatment is more down-toearth.

\section{Notation}

0.1) Let $R$ be a ring (all rings are assumed to be commutative and have the unit element). We denote by $R\left[\left[x_{1}, \cdots, x_{n}\right]\right]=R[[x]]$ the power series ring in $n$ variables over $R . R[[x]]_{0}$ is the set of elements $f(x) \in R[[x]]$ with $f(0)=0$, and $R[[x]]_{0}^{m}$ is the additive group of vectors $\left(f_{1}(x), \cdots, f_{m}(x)\right)$ with $f_{i}(x) \in R[[x]]_{0}, 1 \leqslant i \leqslant m$. Let

$$
\begin{aligned}
& f=\left(f_{1}, \cdots, f_{m}\right) \in R\left[\left[x_{1}, \cdots, x_{n}\right]\right]_{0}^{m} \text { and } \\
& g=\left(g_{1}, \cdots, g_{l}\right) \in R\left[\left[x_{1}, \cdots, x_{m}\right]\right]_{0}^{l} .
\end{aligned}
$$

We denote by $g \circ f$ the element $h=\left(h_{1}, \cdots, h_{l}\right)$ of $R\left[\left[x_{1}, \cdots, x_{n}\right]\right]_{0}^{l}$ where

$$
h_{i}=g_{i}\left(f_{1}, \cdots, f_{m}\right), 1 \leqslant i \leqslant l .
$$

If $l=m=n$ and

$$
\begin{aligned}
& f \circ g\left(x_{1}, \cdots, x_{n}\right)=\left(x_{1}, \cdots, x_{n}\right) \\
& g \circ f\left(x_{1}, \cdots, x_{n}\right)=\left(x_{1}, \cdots, x_{n}\right),
\end{aligned}
$$

we say $f$ is invertible and denote $g$ by $f^{-1}$.

$I d \in R[[X]]_{0}^{n}$ is defined by

$$
I d\left(X_{1}, \cdots, X_{n}\right)=\left(X_{1}, \cdots, X_{n}\right) .
$$

Received May 8, 1970. 
0.2) Let $R$ be a complete local Noetherian ring, with maximal ideal $m$ and residue field $k$ such that char. $k=p>0 . \quad C=\boldsymbol{C}_{R}$ is the category of Artinian local $R$-algebras having the residue field $k$. All homomorphisms are assumed to be local. Let $\hat{\boldsymbol{C}}=\hat{\boldsymbol{C}}_{R}$ denote the category of complete Noetherian local $R$-algebras with residue field $k$.

0.3) Let $A$ be a ring and $F=\left(F_{1}, \cdots, F_{n}\right)$ a formal group over $A$. All formal groups are assumed to be commutative. Sometimes it is convenient to regard $F$ as a group functor from the category of $A$-algebra to the category of sets:

$$
\begin{aligned}
F(B)= & \left\{\left(b_{1}, \cdots, b_{n}\right) \mid b_{i} \in B\right. \text { nilpotent } \\
& \left.\left(b_{1}, \cdots, b_{n}\right)+\left(b^{\prime}{ }_{1}, \cdots, b_{n}^{\prime}\right)=\left(F_{1}\left(b, b^{\prime}\right), \cdots, F_{n}\left(b, b^{\prime}\right)\right)\right\} .
\end{aligned}
$$

0.4) Definition 1. Let $\Phi$ be a formal group over $k$ and $F$ a formal group over $S \in \hat{C} . \quad F$ is a deformation of $\Phi$ over $S$ if the reduction $\bar{F}$ of $F$ is $\Phi$.

Morphisms of the deformations are morphisms of formal groups whose reductions are the identity $I d$.

We define the functor $D$ for which $D(S)$ is the set of the isomorphism classes of the deformations of $\Phi$ over $S$.

\section{Lifting of $p$-divisible groups}

Let $A^{\prime} \stackrel{p^{\prime}}{\longrightarrow} A, A^{\prime \prime} \stackrel{p^{\prime \prime}}{\longrightarrow} A$ be morphisms in $C$. There is a natural map

$$
D\left(A^{\prime} X_{A} A^{\prime \prime}\right) \longrightarrow D\left(A^{\prime}\right) X_{D(A)} D\left(A^{\prime \prime}\right) .
$$

1.1) Lemma 1. (*) is surjective if $A^{\prime \prime} \stackrel{p^{\prime \prime}}{\longrightarrow} A$ is surjective.

Proof. Let $F^{\prime} \in D\left(A^{\prime}\right), F^{\prime \prime} \in\left(A^{\prime \prime}\right)$ and $F^{\prime} X F^{\prime \prime} \in D\left(A^{\prime}\right) X_{D(A)} D\left(A^{\prime \prime}\right)$. We denote by $F_{\left.\right|_{A}}$ (resp. $\left.F^{\prime \prime}\right|_{A}$ ) the reduction of $F^{\prime}\left(\operatorname{resp} . F^{\prime \prime}\right)$ to $A$. Then, there is an isomorphism $\bar{\alpha}:\left.\left.F^{\prime}\right|_{A} \longrightarrow F^{\prime \prime}\right|_{A}$ such that the reduction of $\bar{\alpha}$ to $k$ is the identity $I d$. Since $p^{\prime \prime}$ is surjective, we can lift $\bar{\alpha}$ to $A^{\prime \prime}$. Let $\alpha$ be a lifting. Define $G(x, y)=\alpha F^{\prime \prime}\left(\alpha^{-1}(x), \alpha^{-1}(y)\right)$. Then $G$ is a deformation of $\Phi$ over $A^{\prime \prime}$ and $G \simeq F^{\prime \prime}$. Let $F(x, y)=\sum a_{\alpha \beta}^{\prime} x^{\alpha} y^{\beta}, G(x, y)=\sum a_{\alpha \beta}^{\prime \prime} x^{\alpha} y^{\beta}$. We set $H(x, y)=$ $\Sigma\left(a_{\alpha \beta}^{\prime}, a_{\alpha \beta}^{\prime \prime}\right) x^{\alpha} y^{\beta}$. Then $H(x, y)$ is a formal group over $A^{\prime} X_{A} A^{\prime \prime}$. Moreover it is a deformation of $\Phi$ and mapped to $F^{\prime} X F^{\prime \prime}$. 
1.2) Lemma 2. Let $\Phi$ be $p$-divisible (for the definition see Tate (4)) and $F$ a deformation of $\Phi$ over $A \in \boldsymbol{C}$.

Then an automorphism $\varphi: F \longrightarrow F$ whose reduction to $k$ is the identity is itself the identity.

Proof. Let $m$ be the maximal ideal of $A$. There exists an integer $n$ such thet $m^{n}=0, m^{n-1} \neq 0$. We prove lemma 2 by induction on $n$. If $n=1$, there is nothing to prove. We assume lemma 2 for $m \leqslant n-1$ and we shall prove lemma 2 holds for $n$. By induction assumption $\varphi \equiv I d \bmod m^{n-1}$. Let $\rho$ be the difference of $\varphi$ and $I d$ in $\operatorname{Hom}(F, F)$, then $\rho$ is an endomorphism of $F$ and all the ceofficients of $\rho$ are in $m^{n-1} \cdot \rho(F(x, y))=F(\rho(x)$, $\rho(y))=\rho(x)+\rho(y)$, since $\left(m^{n-1}\right)^{2}=m^{2 n-2} \subset m^{n}$. On the other hand $m^{n-1} / m^{n}$ is a vector space over $k$. Therefore, $\rho \Phi([p] x)=p \cdot \rho(x)=0$. As $\Phi$ is $p$-divisible, $\rho=0$. It follows that $\varphi=I d$.

1.3) Lemma 3. If $p^{\prime \prime}$ is surjective and $\Phi$ is $p$-divisible, then $(*)$ is bijective.

Proof. Let $F^{\prime} X F^{\prime \prime} \epsilon D\left(A^{\prime}\right) X_{D(A)} D\left(A^{\prime \prime}\right)$. As in the proof of lemma 1, we may assume $\left.F^{\prime}\right|_{A}=\left.F^{\prime \prime}\right|_{A}=F$, where $\left.F^{\prime}\right|_{A}$ (resp. $\left.F^{\prime \prime}\right|_{A}$ ) is the reduction of $F^{\prime}$ (resp. $F^{\prime \prime}$ ) to $A$. Let $G$ be a formal group constructed in the proof of lemma 1. Let $H$ be another formal group over $A^{\prime} X_{A} A^{\prime \prime}$ with the reductions $H_{\mid A^{\prime}} \stackrel{\varphi^{\prime}}{\longrightarrow} F^{\prime}, H_{\left.\right|_{A \prime} \prime} \stackrel{\stackrel{\varphi^{\prime \prime}}{\longrightarrow}}{\longrightarrow} F^{\prime \prime}$. Since the projection $A^{\prime} X_{A} A^{\prime \prime} \longrightarrow A^{\prime}$ is surjective, we may assume $\varphi^{\prime}=I d$. We sahll show the reduction $\varphi_{A}^{\prime \prime}=I d$. In fact $H_{\mid A}=\left.F \stackrel{\varphi_{1 A}^{\prime \prime}}{\longrightarrow} F^{\prime \prime}\right|_{A A}=F$ induces an automorphism of the deformation $F$. By lemma $\left.2 \varphi^{\prime \prime}\right|_{A}=I d$. This shows $\varphi^{\prime} X \varphi^{\prime \prime} \in A^{\prime} X_{A} A^{\prime \prime}[[x]]$ and $\varphi^{\prime} X \varphi^{\prime \prime}$ gives an isomorphism $H \stackrel{\longrightarrow}{\longrightarrow}$.

\section{Cohomology group of a formal group}

2.1) Definition 2. Let $R$ be a ring and $M$ an $R$-module. We denote by $M\left[\left[x_{1}, \cdots, x_{n}\right]\right]_{0}=M[[x]]_{0}$ the submodule of $M \otimes_{R} R\left[\left[x_{1}, \cdots, x_{n}\right]\right]$ generated by the elements without constant term (cf. Lubin and Tate (1)).

We define the cohomology group of a formal group as in Lubin and Tate (1).

Definition 3. Let $F(x, y) \in R\left[\left[x_{1}, \cdots, x_{n}, y_{1}, \cdots, y_{n}\right]\right]^{n}=R[[x, y]]^{n}$ be a formal group of dimension $n$. If $f \in M\left[\left[x_{1}, \cdots, x_{n}\right]\right]_{0}^{n}$, then $\delta_{F} f \in M\left[\left[x_{1}, \cdots x_{n}\right.\right.$, $\left.\left.y_{1}, \cdots, y_{n}\right]\right]_{0}^{n}$ is defined by 


$$
\delta_{F} f(x, y)=f(y)-f(F(x, y))+f(x)
$$

If $\left.f \in M\left[\left[x_{1}, \cdots, x_{n}, y_{1}, \cdots, y_{n}\right]\right]_{0}^{n}=M[x, y]\right]_{0}^{n}$, then

$$
\delta_{F} f \in M\left[\left[x_{1}, \cdots, x_{n}, y_{1}, \cdots, y_{n}, z_{1}, \cdots, z_{n}\right]\right]_{0}^{n}=M[[x, y, z]]_{0}^{n}
$$

is defined by

$$
\delta_{F} f(x, y, z)=f(y, z)-f(F(x, y), z)+f(x, F(y, z))-f(x, y) .
$$

$B_{M}^{2}(F)$ is the set of all $f \in M[[x, y]]_{0}^{n}$ such that $f=\delta g$ for some $g \in M[[x]]_{0}^{n}$. $Z_{M}^{2}(F)$ is the set of all $f \in M[[x, y]]_{0}^{n}$ such that $\delta f=0$ and $f(x, y)=f(y, x)$. $H_{M}^{2}(F)$ is defined by $Z_{M}^{2}(F) / B_{M}^{2}(F)$.

2.2) Let $R$ be a ring, $J$ an ideal of $R$ such that $J^{2}$ is the zero ideal and $F$ a formal group over $R$. We denote by $\bar{F}$ the reduction of $F$ to $R / J$. A formal group $G$ over $R$ having $\bar{F}$ as the reduction is called a deformation of $\bar{F}$.

Proposition 1. There is 1-1 correspondence between the set of isomorphism classes of deformations of $F$ and the elements of the group $H_{J}^{2}(F)$.

The equivalence of two deformatinos is defined as in 0.4) definition 1. Before we proceed to the proof, we give some remarks and simplify the notation. Let $u, v$ be elements of $R[[x]]_{0}^{n}$. We denote by $u * v$ the vector $F(u, v) \in R[[x, y]]_{0}^{n}$. We denote by $\frac{1}{u}$ the vector $i(u)$ such that $F(u, i(u))=0$. + is the usual addition of vectors. Since $J^{2}=(0), u * v=u+v$ if all the coefficients of $u$ and $v$ are in $J$.

Proof of proposition. Let $G$ be a formal group over $R$ such that the reduction is $\bar{F}$. We define $d(F, G) \in J[[x, y]]_{0}^{n}$ by $\frac{1}{F} * G$. We show that $d(F, G)$ is a cocycle. Since the coefficients of $d(F, G)$ are in $J$, by the remark above,

$$
\begin{aligned}
& \delta(d(F, G))(x, y, z)=d(F, G)(y, z)-d(F, G)(x * y, z)+d(F, G)(x, y * z) \\
&-d(F, G)(x, y) \\
&=d(F, G)(y, z) * \frac{1}{d(F, G)(x * y, z)} * d(F, G)(x, y * z) * \frac{1}{d(F, G)(x, y)} \\
&=\frac{1}{z} * G(y, z) * \frac{1}{G(x * y, z)} * G(x, y * z) * x * \frac{1}{G(x, y)} .
\end{aligned}
$$

To show this is equal to zero, it is sufficient to prove, 


$$
\begin{aligned}
& \frac{1}{x} * G(x * y, z)=\frac{1}{G(x, y)} * G(G(x, y), z) * y, \\
& \frac{1}{z} * G(x, y * z)=\frac{1}{G(y, z)} * G(x, G(y, z)) * y .
\end{aligned}
$$

In fact, since the coefficients of $d(F, G)$ are in $J$ and $J^{2}=(0)$,

$$
d(F, G)(x * y, z)=d(F, G)(G(x, y), z) .
$$

By definition,

$$
\begin{aligned}
& \frac{1}{x} * \frac{1}{y} * \frac{1}{z} * G(x * y, z)=\frac{1}{G(x, y)} * \frac{1}{z} * G(G(x, y), z) \\
& \frac{1}{x} * \frac{1}{y} * G(x * y, z)=\frac{1}{G(x, y)} * G(G(x, y), z) \\
& \frac{1}{x} * G(x * y, z)=\frac{1}{G(x, y)} * G(G(x, y), z) * y
\end{aligned}
$$

The other equation follows from

$$
d(F, G)(x, y * z)=d(F, G)(x, G(y, z)) .
$$

Let $G$ be a formal group such that the reduction $\bar{G}$ is $\bar{F}$. We show $G$ is equivalent to $F$ if and only if $d(F, G)$ is a coboundary. In fact $G$ is equivalent to $F$ if and only if there exists $u^{\prime} \in R[[x]]_{0}^{n}$ such that $F\left(u^{\prime}(x), u^{\prime}(y)\right)$ $=u^{\prime}\left(G(x, y)\right.$. Define $u$ by $\frac{1}{I d} * u=u^{\prime}$, then

$$
\begin{aligned}
& F\left(\frac{1}{x} * u(x), \frac{1}{y} * u(y)\right)=\frac{1}{G(x, y)} * u(G(x, y)) \\
& \frac{1}{F(x, y)} * u(x) * u(y)=\frac{1}{G(x, y)} * u(G(x, y)) \\
& \frac{1}{F(x, y)} *(u(x)+u(y))=\frac{1}{G(x, y)} * u(G(x, y)) \\
& G(x, y) * \frac{1}{F(x, y)} *(u(x)+u(y))=u(G(x, y)) \\
& d(F, G)(x, y)+u(x)+u(y)=u(G(x, y)) \\
& d(F, G)(x, y)=-u(x)+u(G(x, y))-u(y) \\
& d(F, G)(x, y)=-u(x)+u(x * y)-u(y)
\end{aligned}
$$

since $J^{2}=(0), u(G(x, y))=u(F(x, y))$.

This shows that $G$ is equivalent to $F$ if and only if $d(F, G)$ is a coboundary. Let $G_{1}, G_{2}$ be formal groups over $R$ such that the reductions $\bar{G}_{1}, \bar{G}_{2}$ are $\bar{F}$. 
Then similarly we can show that $G_{1}$ is equivalent to $G_{2}$ if and only if $d\left(F, G_{1}\right)-d\left(F, G_{2}\right)$ is a coboundary. It follows that the map

Deformations of $\bar{F} \longrightarrow H_{J}^{2}(\bar{F})$

$$
G \longmapsto d(F, G)
$$

is injective.

For surjectivity, we have to show that for any cocycle $d$ there exists a deformation $G$ of $F$ and a 1-cochain $u$ such that

$$
G=d * F * \delta u \text {. }
$$

For any 1-cochain $u \quad G=d * F * \delta u$ is already commutative and associative. We only have to show that there exists $u$ such that $G(0, x)=0$. It suffices to put $u(x)=d(0, x)$.

\section{Schlessinger's criterion}

3.1) Theorem (Schlessinger). Let $F$ be a (covariant) functor from $\boldsymbol{C}$ to the category of sets (Sets) such that $F(k)=$ one point. Let $A^{\prime} \longrightarrow A$ and $A^{\prime \prime} \longrightarrow A$ be morphisms in $\boldsymbol{C}$. Consider the map

$$
F\left(A^{\prime} X_{A} A^{\prime \prime}\right) \longrightarrow F\left(A^{\prime}\right) X_{F(A)} F\left(A^{\prime \prime}\right) .
$$

Then $F$ is pro-representable (i.e. there exists $T \in \hat{C}$ such that $F(S)=\operatorname{Hom}_{R}(S, T)$ ) if and only if $\left(H_{1}\right),\left(H_{2}\right),\left(H_{3}\right)$ and $\left(H_{4}\right)$ are satisfied

$\left(H_{1}\right) \quad(* *)$ is surjective whenever $A^{\prime \prime} \longrightarrow A$ is a small extention. (see Schlessinger (3))

$\left(H_{2}\right) \quad(* *)$ is bijective when $A=k$ and $A^{\prime \prime}=k[\varepsilon]$, with $\varepsilon^{2}=0$.

REMARK. If $\left(H_{2}\right)$ is satisfied, $F(k[\varepsilon])$ has a natural $k$-vector space structure (see Schlessinger (3)).

$\left(H_{3}\right) \quad \operatorname{dim} F(k[\varepsilon])<\infty$

$\left(H_{4}\right) \quad$ For any smalll extension $A^{\prime} \longrightarrow A$

$$
F\left(A^{\prime} X_{A} A^{\prime}\right) \longrightarrow F\left(A^{\prime}\right) X_{F(A)} F\left(A^{\prime}\right)
$$

is bijective.

3.2) Theorem 1. The functor $D$ from $\hat{C}$ to (Sets) is representable if $k$ is perfect and $\Phi$ is $p$-divisible. 
Proof. To prove $D$ is representable, it is sufficient to show that the restriction of $D$ to the full subcategory $\boldsymbol{C}$ is pro-representable. We shall prove that $D$ satisfies $\left(H_{1}\right),\left(H_{2}\right),\left(H_{3}\right)$ and $\left(H_{4}\right)$. 1.3) Lemma 3 already shows that $\left(H_{1}\right),\left(H_{2}\right)$ and $\left(H_{4}\right)$ are satisfied. Since $\Phi$ has a lifting to $k[\varepsilon], D(k[\varepsilon])=$ $H_{k}^{2}(\Phi)$ by proposition 1 . It is easy to verify that this correspondence is an isomorphism of vector spaces. On the other hand the additive group functor $G_{a}$ from the category of $k$-algebras is smooth (i.e. $G_{a}(A) \longrightarrow G_{a}(A / I)$ is surjective if $I$ is a nilpotent ideal) and consequently every extension has a functorial section. Hence $H_{k}^{2}(\Phi) \simeq \operatorname{Ext}\left(\Phi, G_{a}^{n}\right)$, where $\operatorname{Ext}$ is the set of extensions of group functors over $k$. To calculate $\operatorname{Ext}\left(\Phi, G_{a}^{n}\right)$, we can use the contravariant Dieudonné module since $k$ is perfect (see Oda (2)). The following calculation is essentially due to Oda. For a formal group $G$, let $M(G)$ denote the corresponding Dieudonné module.

Then $M\left(G_{a}\right)=A / A V$. We put $M(\Phi)=N$. From the exact sequence

$$
0 \longrightarrow A \underset{\text { right multiplication by } V}{\longrightarrow} A \longrightarrow A / A V \longrightarrow 0
$$

we get an exact sequence

$$
\begin{aligned}
& 0 \longrightarrow \operatorname{Hom}(A \mid A V, N) \longrightarrow \operatorname{Hom}(A, N) \longrightarrow \operatorname{Hom}(A, N) \\
& \longrightarrow \operatorname{Ext}(A \mid A V, N) \longrightarrow \operatorname{Ext}(A, N) .
\end{aligned}
$$

On the other hand $\operatorname{Hom}(A / A V, N)=\operatorname{Ext}(A, N)=0, \operatorname{Hom}(A, N)=N$ and the map $\operatorname{Hom}(A, N) \longrightarrow \operatorname{Hom}(A, N)$ is the left multiplication by $V$. This implies $\operatorname{dim} \operatorname{Ext}(A \mid A V, N)=\operatorname{corank}(\Phi) . \quad$ Consequently $\operatorname{dim}_{k} \operatorname{Ext}\left(\Phi, G_{a}^{n}\right)=n \operatorname{corank}(\Phi)$. This completes the proof of Theorem 1.

\section{References}

[1] J. Lubin and Tate: Formal moduli for one-parameter Lie groups. Bull. Soc. Math. France 94, 1966.

[2] T. Oda: Abelian Varieties over a Perfect Field and Dieudonné Modules, Ann. Sc. Ecole Norm. Sup. Paris, 2, 1969.

[ 3 ] M. Schlessinger: Functors of Artin rings. Trans. Amer. Soc. 130, 1968.

[4] J. Tate: p-divisible groups, in Local Fields, Springer-Verlag, 1967.

Nagoya University 